\title{
Power Consumption Evaluation of Circuit-Switched Versus Packet-Switched Optical Backbone Networks
}

\author{
Ward Van Heddeghem, Bart Lannoo, \\ Didier Colle and Mario Pickavet \\ Ghent University-iMinds, Ghent, Belgium \\ Email: ward.vanheddeghem@intec.ugent.be
}

\author{
Francesco Musumeci \\ and Achille Pattavina \\ CNIT and Politecnico di Milano, \\ Milan, Italy \\ Email: fmusumeci@elet.polimi.it
}

\author{
Filip Idzikowski \\ Technische Universität Berlin, TKN, \\ Berlin, Germany \\ Email: filip.idzikowski@tu-berlin.de
}

\begin{abstract}
While telecommunication networks have historically been dominated by a circuit-switched paradigm, the last decades have seen a clear trend towards packet-switched networks. In this paper we evaluate how both paradigms perform in optical backbone networks from a power consumption point of view, and whether the general agreement of circuit switching being more power-efficient holds. We consider artificially generated topologies of various sizes, mesh degrees and-not yet previously explored in this context-transport linerates. We cross-validate our findings with a number of realistic topologies.

Our results show that, as a generalization, packet switching can become preferable when the traffic demands are lower than half the transport linerate. We find that an increase in the network node count does not consistently increase the energy savings of circuit switching over packet switching, but is heavily influenced by the mesh degree and (to a minor extent) by the average link length.
\end{abstract}

\section{INTRODUCTION}

Electricity consumption in telecommunication networks is an important issue — The worldwide electricity consumption of telecommunication networks (which includes operator networks, office network equipment, and customer premises network equipment) has been estimated to be $350 \mathrm{TWh}$ in 2012 , accounting for $1.8 \%$ of the total worldwide electricity consumption in the same year [1]. While it can be argued that this number in itself is relatively small, it is non-negligible and increasing at a rate of $10 \%$ per year. Moreover, its relative contribution to the total worldwide electricity consumption is increasing as well (from $1.3 \%$ in 2007 to $1.8 \%$ in 2012). As such, the interest to improve the energy-efficiency of telecommunication networks is a hot research topic, and is of importance for economic (reducing the energy cost), technical (reducing the associated heat dissipation) and environmental (reducing the carbon footprint) reasons.

The electricity consumption in backbone networks is expected to rise considerably — The major part of the power consumption in the telecommunication operator networks is currently attributed to the wired aggregation \& access network and mobile radio network. The backbone network, in contrast, is estimated to account (in 2012) for only about $8 \%$ of the total operator network consumption (which includes the wired aggregation \& access, mobile radio and backbone network) [2]. However, the energy consumption in wired access networks is proportional to the number of connected subscribers, while the consumption in the backbone network is proportional to the traffic volume [2]. With the expected increase of traffic volume, high growth rates in the backbone's energy consumption are expected (potentially even overtaking the access network's consumption [3]). For this reason, it is important to react timely to the energy issue of backbone networks.

In this paper we extensively compare the circuit and packetswitched Internet Protocol (IP)-over-Wavelength Division Multiplexing (WDM) networks with respect to their power efficiency. We consider circuit switching in the context of optical circuits, in contrast to the more traditional opto-electronic circuit switching such as in SONET/SDH and OTN.

Earlier work on the power efficiency of circuit vs packet switching - In the last decades, the telecommunication industry has seen a shift from circuit-switched networks to packet-switched networks. We focus on the comparison of circuit switching and packet switching in terms of (inverse) power efficiency, leaving the more complex hybrid solutions aside. There has been some earlier research into the power consumption of circuit switching versus packet switching, identified respectively as bypass and non-bypass architectures in the context of optical networks. In [4] the authors exploited the concept of lightpath-bypass to perform a power-minimized optical network design, based on Integer Linear Programming (ILP) formulations and heuristics. They distinguish non-bypass (packet switching), direct bypass (circuit switching), as well as an intermediate hybrid solution called multi-hop bypass. A similar problem has been faced in our previous work [5], where simulations and an analytical model were used for the power consumption evaluation of bypass and non-bypass scenarios. In the line of these studies, an analytical model based on expectation values has been also developed in [6], where different variations of the optical bypass strategy are evaluated under different mesh degree scenarios, i.e., from a ring up to full-mesh topologies. Capital Expenditure (CapEx) minimized and power minimized networks designed with an ILP and a genetic algorithm have been considered in our previous work [7]. A bypass and non-bypass architectures (differing by traffic grooming, placement of transponders and (non-)existence of Optical Cross-Connects (OXCs)) in IP-overWDM are distinguished. Finally, in [8], a power consumption evaluation of switching and routing elements is performed to 


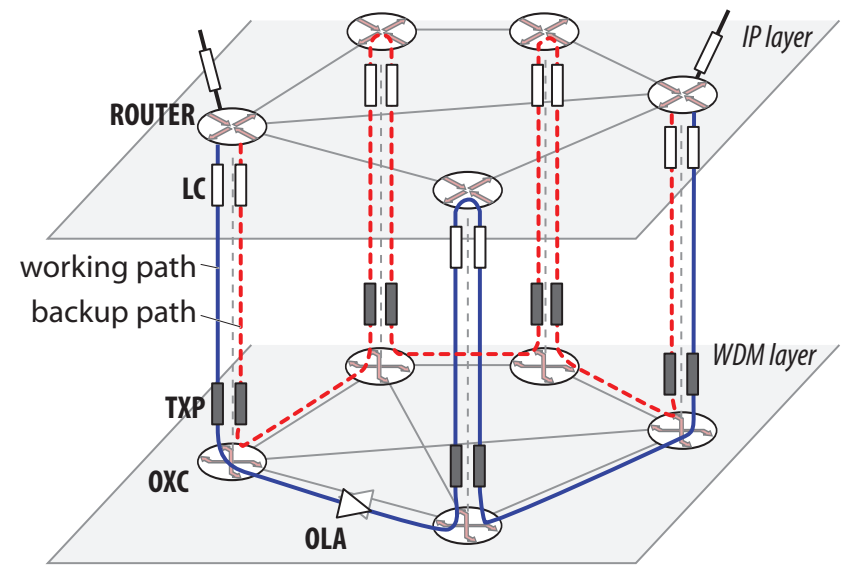

(a) Packet switching

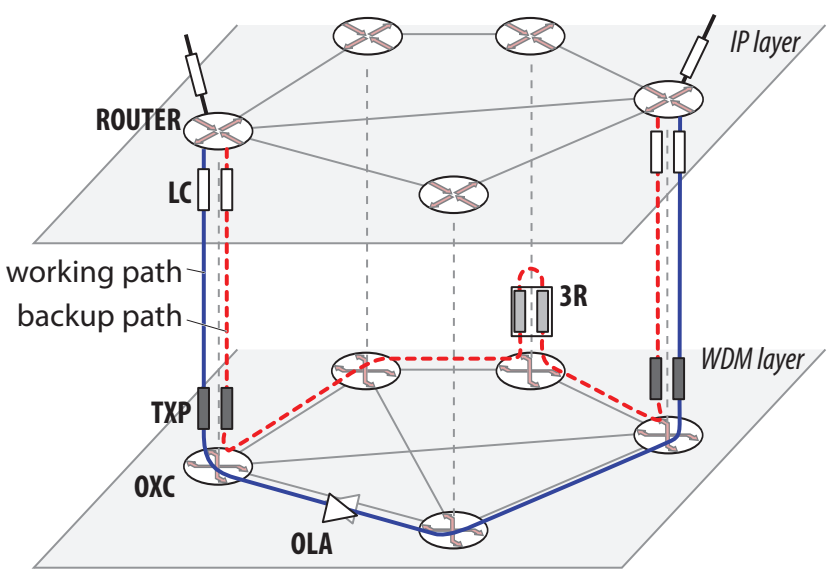

(b) Circuit switching

Fig. 1. The packet-switched and circuit-switched network architectures considered in this paper, showing both the bidirectional working path (solid lines) and backup path (dashed lines) under a $1+1$ protection scheme. $(\mathrm{LC}=$ line card, TXP $=$ transponder, OXC $=$ Optical Cross-Connect, OLA $=$ Optical Line Amplifier, $3 \mathrm{R}=3 \mathrm{R}$ regenerator)

compare the circuit and packet switching paradigms, but the analysis is limited to the node level.

The works mentioned above point out the benefits of circuit switching over packet switching in terms of power consumption. These benefits depend however on the investigated network scenario. For example, looking at Fig. 4 of [4], the $\mathrm{x}$-axis depicting "Average of random traffic demand" starts from $20 \mathrm{Gbps} /$ node pair, while the capacity of a single WDM channel is set to $40 \mathrm{Gbps}$. The missing range $0-20 \mathrm{Gbps} /$ node is expected to show that the packet-switched networks can be less power consuming than the circuit-switched networks, as preliminarily indicated in [5] and [7].

Contributions of this paper - The four key contributions of our paper with respect to the existing research are as follows. (1) In addition to considering the mesh degree and network size (in terms of the number of nodes and average physical link length), we evaluate the influence of the channel linerate on the power efficiency of circuit switching versus packet switching, a parameter which to our knowledge has previously not been assessed. (2) We particularly look at network scenarios where packet switching is preferable from a power consumption point of view. This aspect has to the best of our knowledge not been addressed in the previous literature (as mentioned above, see e.g., lack of the range 0-20 Gbps/node pair of average traffic in the Fig. 4 of [4]). One way in which we do this is by considering the ratio of the average node-tonode demand over the channel linerate. (3) We deeply study the (inverse) power efficiency of both switching paradigms under increasing traffic demand. We show that the power efficiency of packet switching in sparsely-connected networks is almost independent of the traffic demand, whereas for circuit switching the power efficiency improves with increasing traffic. (4) We find that a higher node count does not necessarily make circuit switching more preferable. In highly meshed networks the node count does not influence the relative savings of circuits switching over packet switching at all. Our results show that the mesh degree, the demand/linerate ratio and the physical link length are critical parameters.

All in all, our results provide a better insight into the tradeoff of the power efficiency of circuit switching versus packet switching.

Organization of this paper - After outlining the network architecture and power consumption model that we use (Section II), we consider both a set of realistic and artificially generated but regular topologies of different sizes (in terms of nodes), different connectivity degree, and various linerates (Section III). Using the result from our dimensioning tool, we show in Section IV that (a) indeed packet switching can be the preferable option with respect to power consumption below certain traffic demand bitrates, (b) that this crossover point is essentially determined by the ratio of the traffic demand over the linerate, and (c) to a minor extent also by the mesh degree.

\section{NETWORK ARCHITECTURE AND POWER CONSUMPTION MODEL}

\section{A. Network architecture}

The general architecture of the network is shown in Fig. 1 on an example of a 5-node topology (IP/Multiprotocol Label Switching (MPLS) and WDM layers). In the IP/MPLS layer, a core router is equipped with line cards, providing one or more ports with short reach interfaces. We assume (differently from [8]) that IP routers have to be present in the backbone network under the circuit switching paradigm, since they exchange the IP traffic with other networks (metro, access) attached to them [9]. The buffers located in the router's line cards are used only at the end nodes of the optical circuits. The granularity of the linerates of the interfaces differs: the access or client-side traffic connects to the router using 1-Gbps interfaces, and the core network side interfaces are either 2.5-Gbps, 10-Gbps or 40 -Gbps interfaces (which we refer to as $2.5 \mathrm{G}, 10 \mathrm{G}$ and $40 \mathrm{G}$ ). Note that, depending on the traffic demand bitrate, one or more interfaces can be required per demand. 
In the WDM layer, long reach transponders with the same capacity as the IP/MPLS layer line cards provide a WDM optical signal, which is switched using an OXC towards the correct physical link. A mux/demux (included in the OXC) aggregates up to 40 channels on a fiber. For each physical link, we assume an unlimited number of fibers to be available. A booster and pre-amplifier (included in the OXC) amplify all channels in a fiber pair respectively upon leaving and entering a node. An Optical Line Amplifier (OLA) is placed every $80 \mathrm{~km}$, and amplifies all channels in a fiber pair. For lightpaths longer than the regenerator span, taken to be $1,500 \mathrm{~km}$, the signal is switched by the OXC to pass through a $3 \mathrm{R}$ regenerator.

The way that traffic demands traverse the network is different in packet switching and circuit switching. Under the packet switching paradigm, all the traffic in a node-i.e., not only the originating and terminating, but also the transit traffic-is processed at the router in the IP/MPLS layer, as shown by the solid line in Fig. 1(a). This provides the opportunity to groom traffic, that is bundling traffic belonging to demands from different sources that are destined to the same outgoing link. As a result, the transport channels (wavelengths) are filled more efficiently.

Under the circuit switching paradigm, traffic demands traverse the network over a single IP hop, since dedicated optical circuits are set up from the source IP/MPLS node to the target node, as shown by the solid line in Fig. 1(b). This allows the transit traffic to remain in the optical domain and thus bypass the IP router. For this reason such architectures are often referred to as optical-bypass architectures. However, depending on the ratio between the traffic demand bitrates and the channel capacity (i.e., linerate), lightpaths might not be optimally used. For a given set of demands, this might result in a higher number of channels required compared to packet switching.

In both switching cases, we assume a $1+1$ protection scheme at the IP layer. Under this scheme, a backup path (dashed line in Fig. 1) is simultaneously routed over a link-disjoint physical path with respect to the primary one, so that if the working path fails, the traffic can be instantaneously switched over to the backup path.

\section{B. Power consumption model}

The power consumption values assumed for each equipment type are listed in Table I. All values are taken from [5], with the exception of the $40 \mathrm{G}$ coherent transponder value which is based on [10].

The power-per-port values for the IP router include both the power consumed by the line card and the basic node (i.e., chassis, switch fabric, routing engine, power supply, internal cooling and remaining minor components). We assume the power-per-port value fixed and independent of the load, as the power consumption of present-day IP routers when idle and under full load are very similar [10], [11]. This also implies that the influence on the power consumption of buffering and table look-up associated with packet switching is negligible. The OXC degree $d_{f}$ is defined as the number of
TABLE I

POWER CONSUMPTION VALUES (SOURCE: [5], [10])

\begin{tabular}{lrr}
\hline Equipment & Power cons. & Inv. pow. eff. \\
\hline IP/MPLS 1G-port & $10 \mathrm{~W}$ & $10 \mathrm{~W} / \mathrm{Gbps}$ \\
IP/MPLS 2.5G-port & $25 \mathrm{~W}$ & $10 \mathrm{~W} / \mathrm{Gbps}$ \\
IP/MPLS 10G-port & $100 \mathrm{~W}$ & $10 \mathrm{~W} / \mathrm{Gbps}$ \\
IP/MPLS 40G-port & $400 \mathrm{~W}$ & $10 \mathrm{~W} / \mathrm{Gbps}$ \\
OLA (per fiber pair, 80 km span) & $110 \mathrm{~W}$ & - \\
Transponder 2.5G & $25 \mathrm{~W}$ & $10 \mathrm{~W} / \mathrm{Gbps}$ \\
Transponder 10G & $50 \mathrm{~W}$ & $5 \mathrm{~W} / \mathrm{Gbps}$ \\
Transponder 40G (coherent) & $167 \mathrm{~W}$ & $4 \mathrm{~W} / \mathrm{Gbps}$ \\
3R regenerator xG & $2 \cdot$ transponder xG & - \\
OXC, 40 ch., with degree $d_{f}$ & $150 \mathrm{~W}+d_{f} \cdot 135 \mathrm{~W}$ & - \\
\hline
\end{tabular}

network-side bidirectional fiber ports, assuming that all fiber ports are added/dropped at the tributary side (i.e., towards the IP/MPLS layer). The power consumption value used for the OXC includes mux/demux stages as well as pre- and boosteramplifiers. In addition to the total power consumed by the devices listed in Table I, we assume that an equal amount of overhead power is consumed for site cooling and power supply losses, i.e., the Power Usage Effectiveness is equal to 2.

\section{CONSIDERED TOPOLOGIES AND POWER CALCULATION METHODOLOGY}

\section{A. Topologies}

To understand the influence of the connectivity degree and network size (in terms of number of nodes and average physical link length) on the power consumption, we consider a number of artificially generated topologies, ranging from minimally meshed (ring) up to maximally meshed (full-mesh) networks, see Table II. To be able to cross-validate our results based on artificial topologies, we also consider three realistic networks: the Spanish Telefónica I+D (TID) network model (forecasted potential topology for the year 2020 [12]), the DICONET pan-European Géant network [13], and the well-known U.S. NSF network [5]. They are also listed in Table II. For all of the networks, the IP topology is taken identical to the WDM topology under the packet switching paradigm. All links are bidirectional. For each of these variations we consider networks with the number of nodes $N$ equal to $10,15,25$, and 33 .

Similarly to [6] we define the mesh degree $M$ of a network as the ratio of the average node degree of the network under consideration, $\bar{d}$, and the node degree of a full-mesh network having the same number of nodes as the considered network, i.e., $d_{m e s h}=\mathrm{N}-1$, so we get $M=\frac{\bar{d}}{d_{\text {mesh }}}$. The half-mesh networks have a mesh degree of $M=0.5$, so that the average desired node degree is easily calculated as $\bar{d}=\frac{N-1}{2}$. To generate these half-mesh networks we (a) start from a ring network with the required number of nodes $N$ and number of links $L_{\text {ring }}=N$, (b) then calculate the number of links to add in order to have the desired ${ }^{1}$ average mesh (and node) degree,

\footnotetext{
${ }^{1}$ Note that, depending on the number of nodes and the requested degree, the theoretical number of links to add might be a fractional number. So we round this value up or down to the closest integer to get a practical (i.e., integral) number of links to add. As a result, the actual degree of the network might differ slightly from the requested one.
} 
TABLE II

TOPOLOGIES CONSIDERED IN THIS STUDY

\begin{tabular}{lrrrrr}
\hline Topology & $\begin{array}{r}\text { Number } \\
\text { of nodes } \\
N\end{array}$ & $\begin{array}{r}\text { Number } \\
\text { of bidir. } \\
\text { links } L\end{array}$ & $\begin{array}{r}\text { Avg. node } \\
\text { degree } \bar{d}\end{array}$ & $\begin{array}{r}\text { Mesh } \\
\text { degree } \\
M\end{array}$ & $\begin{array}{r}\text { Link } \\
\text { length } \\
\text { (avg) } \\
{[\mathrm{km}]}\end{array}$ \\
\hline ring & 10 & 10 & 2 & 0.22 & 255 \\
ring & 15 & 15 & 2 & 0.14 & 166 \\
ring & 25 & 25 & 2 & 0.08 & 100 \\
ring & 33 & 33 & 2 & 0.06 & 75 \\
\hline half-mesh & 10 & 23 & 4.5 & 0.50 & 255 \\
half-mesh & 15 & 53 & 7 & 0.50 & 166 \\
half-mesh & 25 & 150 & 12 & 0.50 & 100 \\
half-mesh & 33 & 264 & 16 & 0.50 & 75 \\
\hline full-mesh & 10 & 45 & 9 & 1.00 & 255 \\
full-mesh & 15 & 105 & 14 & 1.00 & 166 \\
full-mesh & 25 & 300 & 24 & 1.00 & 100 \\
full-mesh & 33 & 528 & 32 & 1.00 & 75 \\
\hline TID & 33 & 53 & 3.21 & 0.10 & $(52.4)$ \\
Géant & 34 & 54 & 3.18 & 0.10 & $(753)$ \\
NSF & 14 & 21 & 3.00 & 0.23 & $(1083)$ \\
\hline
\end{tabular}

and (c) eventually add these links distributed evenly across the ring (connecting the most-distant nodes, based on the hop count, first). Note that the number of links in such a half-mesh network is given by $L=L_{\text {ring }}+\left(N \cdot \frac{\bar{d}-2}{2}\right)=N \cdot \frac{N-1}{4}$.

For the physical link lengths, which influence the power consumption of the OLAs and 3R regenerators, we assume that each of the generated networks covers a geographical area with a diameter of $2,500 \mathrm{~km}$ (which is comparable to a country-sized network). The physical link lengths are then taken to be $2,500 \mathrm{~km}$ divided by the number of links in a ring network. For the half-meshed and full-mesh networks we take all other physical links to have the same length, even if this is topologically unrealistic (Table II).

\section{B. Network dimensioning and power consumption calculation}

We calculate and evaluate the power consumption in the above listed networks for both the packet and circuit-switched architectures, using a custom Java-based dimensioning tool. The general steps in calculating the power consumption are as follows. First, for each topology, a traffic matrix is generated with uniform demands (i.e., an identical demand between each node pair). We also vary the demands bitrate values in different runs. Second, each demand is routed across the network. To achieve 1+1 protection at the IP layer (see Fig. 1), the two shortest link-disjoint physical paths between the source and target nodes are calculated using a minimum cost flow algorithm, where we assume the overall path length, expressed in number of hops, as cost. Third, wavelengths (i.e., channels) and fibers to be used are selected in a first-fit fashion. This means that the algorithm finds the first free wavelength/fiber pair that is available on the physical path between source and target nodes. Fourth, from the node port counts we derive the power consumption of IP/MPLS routers, transponders and OXCs. From the link and path lengths we calculate the number of required OLAs and regenerators, and subsequently their power consumption. Because of the dimensioning tool constraints, we generalized on the $\mathrm{OXC}$ power consumption and calculate an average $\mathrm{OXC}$ power consumption value based on the average node degree of the network.

\section{RESULTS AND OBSERVATIONS}

In this section we compare the power consumption of packet switching (PS) and circuit switching (CS) architectures, evaluated over the artificially generated topologies (from ring to full-mesh) and cross-validated with the realistic topologies.

For this evaluation we use three metrics: the absolute power consumption $(\mathrm{kW})$, the inverse power efficiency (W/Gbps), and the relative power consumption savings of CS over PS (\%).

\section{A. General observations}

Sparser topologies consume more - From Fig. 2(a) and (b) we see that sparser topologies (i.e., more ring-like) consume more power than more meshed topologies. This is due to longer paths needed both in the PS and CS.

Power efficiency improves with increasing demands, except for PS in sparse topologies - Fig. 2(c) and (d) show the inverse power efficiency, i.e., the power (in Watt) required to transport a uniform demand of $1 \mathrm{Gbps}$. We see that the power efficiency of PS (dashed lines) is almost independent of the traffic demand in ring-like networks, whereas in highly-meshed topologies its efficiency starts off worse but gradually improves with increasing traffic. CS (solid lines) behavior is similar to the latter irrespective of the mesh degree.

Higher demands favor $C S$ - For higher traffic demands the PS architecture consumes more power than the CS architecture. On the other hand, for lower traffic the most power-efficient architecture of the two depends on the mesh degree and channel capacity.

\section{B. Influence of the channel linerate $(2.5 G, 10 G, 40 G)$}

Inefficient usage of capacity for higher linerates in CS In Fig. 2(a) and (b) it can be seen that the CS architecture exhibits (i) a very minor linear component (e.g., the CS-10G curve is almost flat between $12 \mathrm{Gbps}$ and $20 \mathrm{Gbps}$ ), due to the fine granularity of the $1 \mathrm{G}$ IP client side ports, and (ii) a much more pronounced stepwise component due to the coarser granularity of the IP network side add/drop ports. The steps occur when the demand bitrate reaches a multiple of the channel linerate $(2.5 \mathrm{G} / 10 \mathrm{G} / 40 \mathrm{G})$. Because of the range of the demand bitrate on the $\mathrm{x}$-axis, the stepwise behavior is most prominent for the $10 \mathrm{G}$ architecture. The overcapacity for the $40 \mathrm{G}$ at such relatively low demands is clear in Fig. 2(b). There is no such stepwise function (or at least, it is much smaller) for PS, because the traffic is groomed, so that the channel capacity (i.e., the linerate) can be used more efficiently even for lower traffic values, and as a result the curve is more linear. As such, the stepwise contribution of CS makes the highercapacity technology (e.g., 40G) much less convenient at low loads. On the other hand, due to the more linear behavior of PS, higher capacity transport technologies such as 10G and $40 \mathrm{G}$ are sooner (i.e., already at low loads) more convenient. 

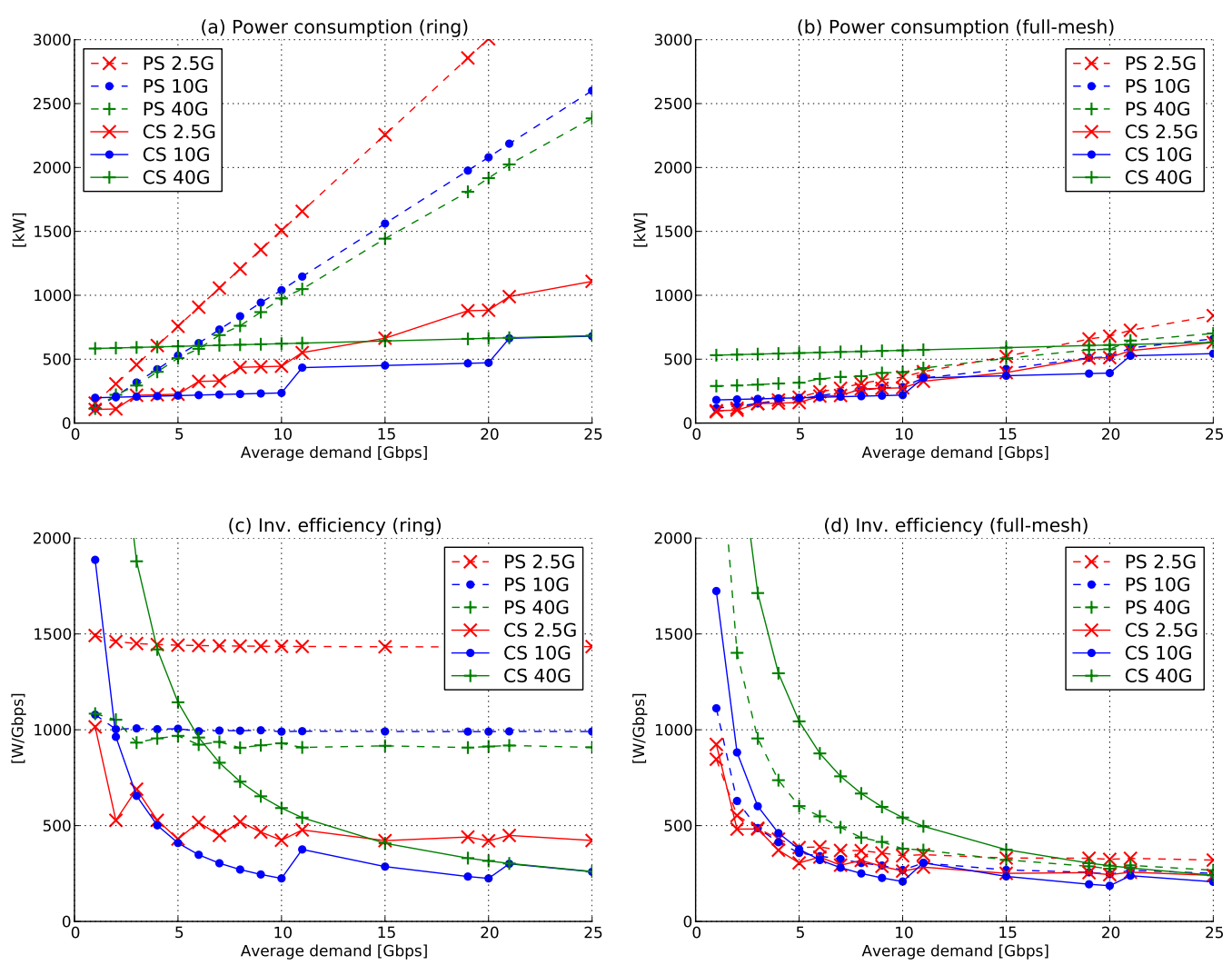

Fig. 2. The total power consumption and inverse power efficiency of a 15-node ring and full-mesh topology with increasing node-to-node traffic demand. The packet-switched (PS) paradigm shows an overall linear behavior, whereas the circuit-switched (CS) paradigm shows a stepwise behavior whenever the traffic demand becomes a multiple of the channel capacity. The power efficiency of PS in sparsely-connected networks is almost independent of the traffic demand, whereas for CS the power efficiency improves with increasing traffic.

Improved power efficiency for higher channel linerates As shown in Table I, the transponders used for the different transport capacities are gradually more power-efficient per Gbps. This is also clearly visible in Fig. 2(c) where the PS $10 \mathrm{G}$ architecture consumes much less power per Gbps than the $2.5 \mathrm{G}$ architecture. The difference between the $10 \mathrm{G}$ and the $40 \mathrm{G}$ architecture is much smaller. The reason for the small increase of power efficiency moving from $10 \mathrm{G}$ to $40 \mathrm{G}$ is because we assumed a coherent $40 \mathrm{G}$ transponder, which comes at an extra power cost.

\section{Influence of the demand/linerate ratio}

To get a clear understanding of when CS is more powerefficient than PS (or vice versa), we plot in Fig. 3 the power consumption savings of CS over PS. Positive values indicate that CS is preferable, negative values indicate that PS is preferable. For a fair comparison between the different channel linerates, we plot this metric against the ratio of the average demand bitrate over the channel linerate. For a ratio equal to 1 , the average demand bitrate is equal to the linerate.

High demand/linerate ratios favor CS, low demand/linerate ratios favor $\mathrm{PS}$ - Fig. 3 shows that increasing demand/linerate ratios lead to higher savings of CS over PS. Low demand/linerate ratios always make PS the preferable paradigm. Both Fig. 3(a) and (b) also clearly show the stepwise behavior around integral multiples of this ratio. Note that the sparse granularity of the data points smooths out the behavior, especially for the $2.5 \mathrm{G}$ architecture (for example, for $2.5 \mathrm{G}$ we have only three data points below the demand/linerate ratio of 1.5 because we consider integer node-to-node demands at 1,2,3, ... Gbps). This behavior originates from the stepwise behavior of the power consumption of the CS architecture. The CS savings increase until the demand reaches the channel capacity (as there is an increasing usage of the channel capacity), and then suddenly drops when the demands surpass the channel capacity (thereby requiring an extra WDM channel).

CS is always preferable for demands higher than half the channel linerate - Fig. 3 also indicates that there is a rather narrow transition window of the demand/linerate ratio where CS becomes more preferable than PS. In sparse networks (Fig. 3(a)) PS is the preferable option up to about demands being $1 / 10$ to $1 / 3$ of the channel linerate. In highly connected networks (Fig. 3(b)), the crossover window is much smaller, and PS is the preferable option for demands being up to half the channel linerate, independently of the utilized transmission technology. The reason that the crossover point is at half the channel linerate is because once a node-to-node demand is larger than half of the channel linerate, there is no free capacity left to groom another demand onto the same channel, and a separate channel is required for each demand. 

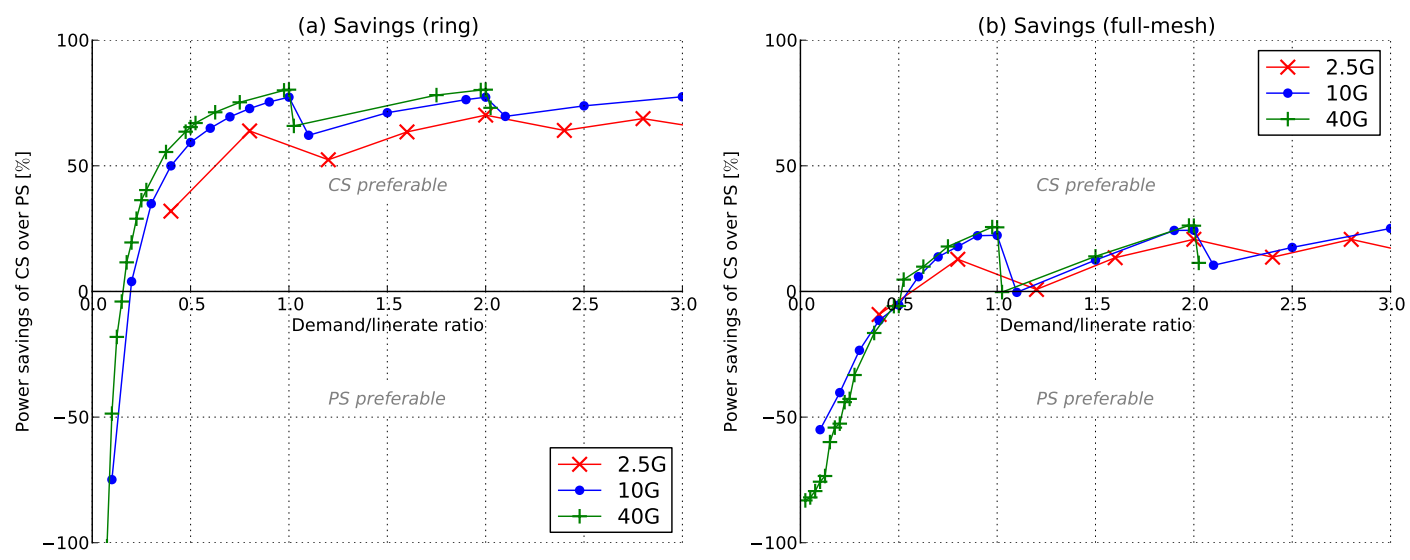

Fig. 3. Power savings of CS over PS mapped to the ratio of the demand bitrate over the channel linerate (15-node topology). The savings show a stepwise behavior around integral multiples of this ratio (i.e, the savings suddenly drop when the node-to-node traffic demands surpass the channel linerate). The ratio's transition window where CS becomes more preferable than PS is relatively small and relatively independent of the channel linerate (especially for highly-meshed networks, where it is fixed at $1 / 2$ ).
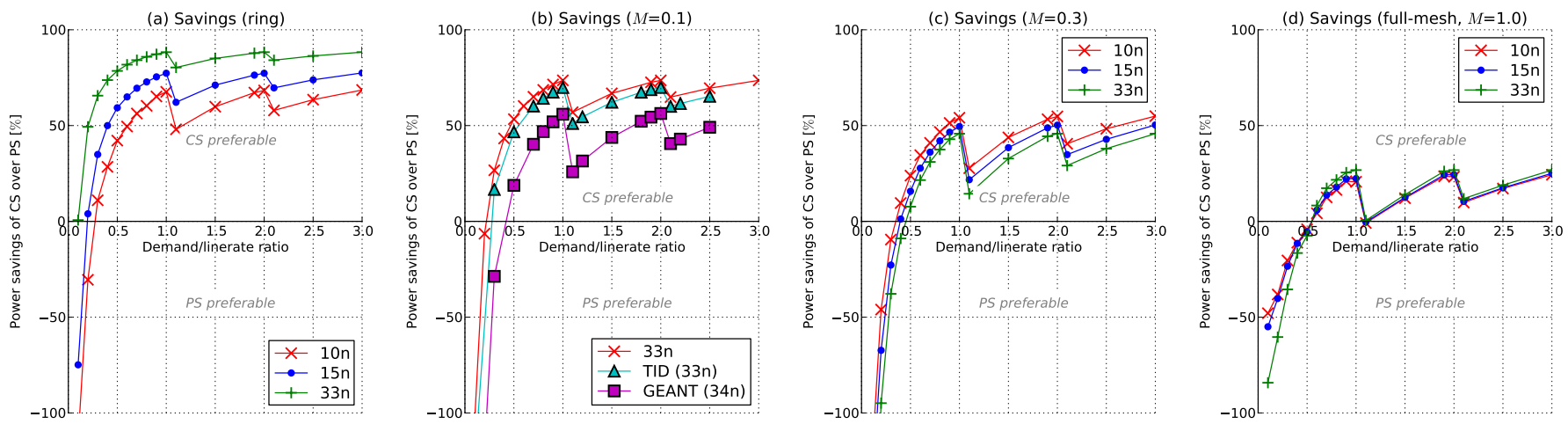

Fig. 4. Influence of the node count on the power savings of CS over PS (for linerate $=10 \mathrm{G}$ ). Only for sparse topologies (i.e, (a) through (c)) the node count has an influence on the savings. While for a ring topology a higher node count leads to more savings, this is not consistently the case for other sparsely meshed topologies. The apparent deviation of the Géant topology from the general trend is explained in Fig. 5.

\section{Influence of the network size (number of nodes and physical link lengths)}

Fig. 4 shows the power consumption savings of CS over PS for networks with different number of nodes (the network with $N=25$ has been omitted for clarity). The subfigures (a) to (d) correspond to an increasing mesh degree. Fig. 4(b) represents a mesh degree $M=0.1$, and contains in addition two realistic topologies that also have $M=0.1$ (the lowest mesh degree of the 10-node and 15-node topology is higher than 0.1, see Table II).

In highly meshed network, the number of nodes does not influence the relative savings - When comparing the four subfigures it is clear that for highly meshed networks, the number of nodes does not influence the relative savings (i.e., all curves coincide in Fig. 4(d)). This is because the IP-layer hop count is low regardless of the number of nodes (the hop count is equal to 1 in full-mesh topologies).

Networks with more nodes do not necessarily result in larger relative savings of CS over PS - For sparse topologies (Fig. 4(a) through (c)) on the other hand the node count has considerable influence on the relative savings of CS over PS. For the ring topology, a higher node count makes CS more preferable. This is due to the higher hop count in larger ring networks, which implies a much higher IP-layer contribution, which increases the PS power consumption. This is inline with [4]. However, our results indicate that the above rule cannot be applied universally to all sparse topologies. In Fig. 4(c) a higher node count does not consistently correspond to increased CS savings (the savings for 33-node artificial topology are lower than for the 15-node topology). Moreover, while in Fig. 4(b) the realistic TID network (33 nodes) savings seems to be inline with the 33-node artificial topology, the Geant network (34 nodes) is certainly not. There must be another parameter with considerable influence at work.

Higher link lengths result in reduced savings for CS - In order to explore the reason of the above described anomaly, Fig. 5(a) plots, in addition to the 33-node artificial topology (physical link length $=75 \mathrm{~km}$ ) and the original Géant topology (average physical link length $=753 \mathrm{~km}$ ), the same Géant topology where all links have been (artificially) set to $75 \mathrm{~km}$. The 

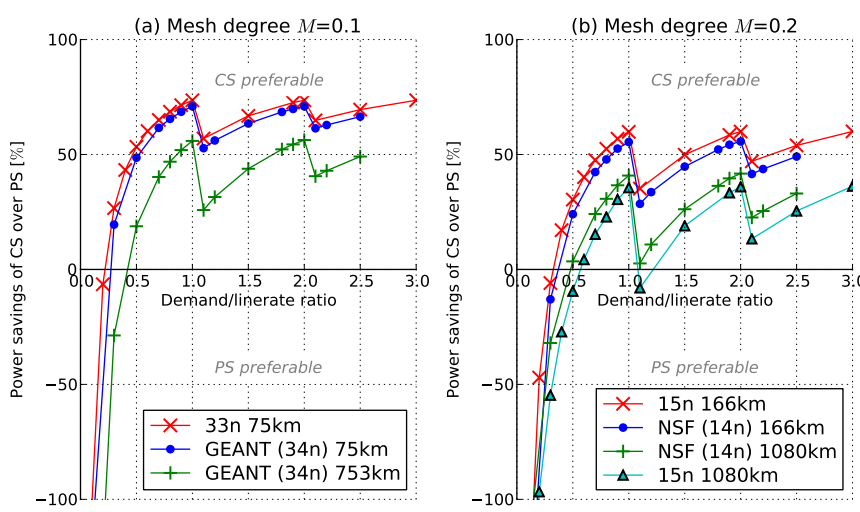

Fig. 5. Influence of the average physical link length on the relative savings of CS over PS (linerate $=10 \mathrm{G}$ ). Higher link lengths result in lower savings, and explain why the savings profile of topologies such as Géant (average physical link length $=753 \mathrm{~km}$ ) does not correspond very well with our artificial topology of the same node count but much lower link length.

figure shows that the difference in link length is the reason of the diverging behavior of the original Géant topology from the artificial 33-node topology. The high link length of the original Géant topology increases the number of required OLAs and $3 \mathrm{R}$ regenerators and the associated power consumption. As the additional power consumption has a larger relative impact on the CS power consumption, the power consumption savings of CS over PS decrease accordingly. This is also confirmed by Fig. 5(b) where the NSF network (14 nodes, mesh degree $M=$ 0.2 , average physical link length $=1080 \mathrm{~km}$ ) is compared with our artificial 15-node $M=0.2$ topology. When the link lengths are adjusted (either from the artificial topology, or from the NSF network), the savings curves become very similar.

\section{E. Influence of the mesh degree}

Although we have not focused on the mesh degree yet, it is already clear from the previous figures and discussion that this parameter is of considerable influence on the power savings

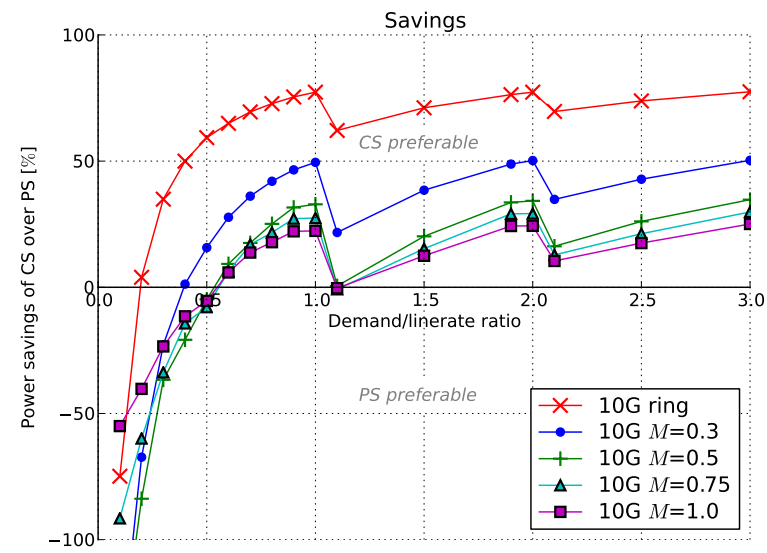

Fig. 6. Influence of the mesh degree on the relative savings of CS over PS (for linerate $=10 \mathrm{G}$, and 15-node topologies). Higher mesh degrees $(M)$ result in lower savings. of CS over PS.

Savings of CS over PS decrease with increasing mesh degree - As shown in Fig. 6, the savings of CS over PS tend to decrease for increasing mesh degree, as adding more edges decreases the hop count and thus more interfaces (i.e., router ports and transponders) can be saved in intermediate nodes of the PS architecture while still performing traffic grooming. On the other hand, for the CS architecture, a higher mesh degree only impacts the OLAs (and eventually, the regenerators) consumption, which constitutes a less relevant contribution in the total consumed power if compared to the power spent by the interfaces.

High channel linerates with low traffic conditions favor PS - An exception to this behavior is obtained for higher channel linerates under low traffic conditions (i.e, low demand/linerate ratios). This is shown in Fig. 7, which plots the savings in function of the mesh degree for different demand/linerate ratios. In this case, passing from ring to half-mesh topologies has, as previously, a higher benefit for the PS than for the CS solution. However, adding further links to the network (i.e., going towards full-mesh topologies), there is lower opportunity for traffic grooming (recall that demands are routed over the two shortest link-disjoint physical paths), so with high channel linerates interfaces are underutilized, thus causing higher relative power consumption.

It is interesting to point out that for the full-mesh case the power consumption of PS and CS are not equal (i.e., CS over PS savings are not zero), as one might incorrectly expect. The link disjoint backup paths always require two hops in both switching paradigms, but the intermediate node requires IP ports under the PS paradigm only, leading to CS being more preferable. However, an exception to this is observed for high linerates (e.g., 40G), combined with low demands bitrate (e.g., 5 Gbps per demand). In this case the opportunity to groom traffic in the PS scenario produces higher power benefits in comparison to the high demands bitrate situation, and thus the CS option is outperformed.

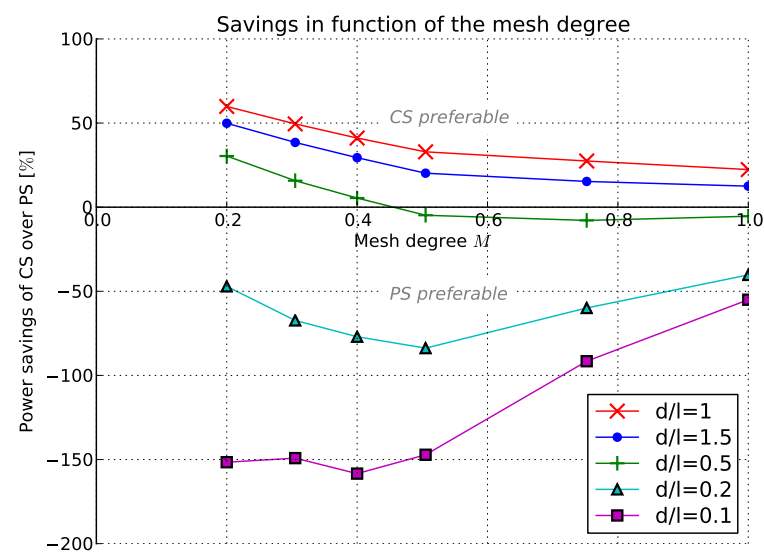

Fig. 7. Influence of the mesh degree and the demand/linerate ratio $d / 1$ on the relative savings of CS over PS (for linerate $=10 \mathrm{G}$, and 15-node topologies). For low demand/linerate ratios there is an optimum point where PS is favorable. 


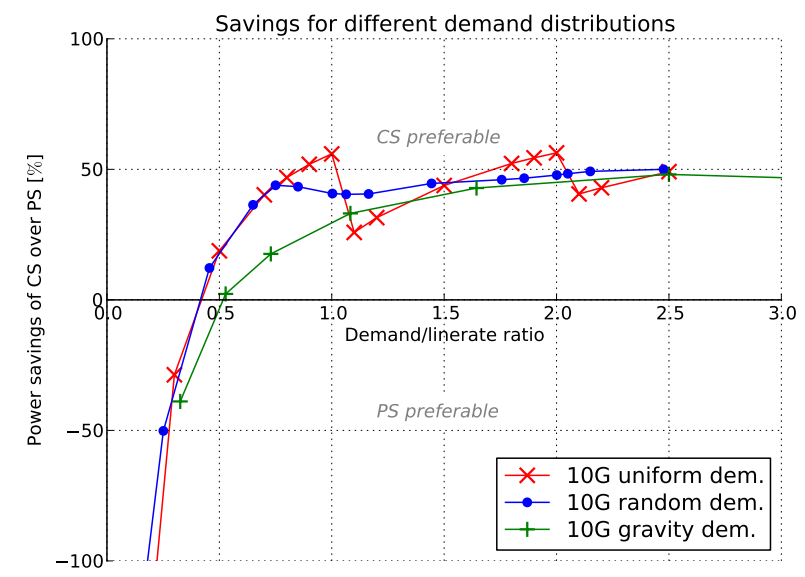

Fig. 8. Influence of different demand types on the savings of CS over PS (Géant topology, linerate $=10 \mathrm{G}$ ). While uniform demands show a distinct stepwise behavior, more realistic demand sets (i.e, random and gravity demands) smooth out this behavior.

\section{F. Sensitivity to non-uniform demands}

In all of the above scenarios we assumed fully-meshed uniform demands. To see the effect of non-uniform demands on the power savings of CS over PS, we consider in Fig. 8 two additional demand types: (a) a gravity traffic matrix where nearby nodes have larger demands, thus closer resembling real life demands [13], and (b) a random fully meshed traffic matrix where each demand is evenly distributed between $-30 \%$ and $+30 \%$ of the nominal demand.

Realistic traffic has a smoother savings profile - While the uniform demands show the distinct step-wise behavior, the behavior is much smoother for random demands and gravity demands. However, the general trend observed before remains valid: CS is preferable for demands higher than half the channel linerate (on average) also under the gravity and random traffic matrices.

\section{CONCLUSION AND FURTHER WORK}

In this paper we extensively compared the power consumption of circuit and packet switching architectures in optical backbone networks. We evaluated the impact of the channel linerate, the network size (both number of nodes and physical link length), demand/linerate ratio and the network mesh degree to assess under which conditions each switching paradigm represents the most power-efficient solution.

We found that, in general, circuit switching is preferable, as fewer IP router ports and WDM transponders are needed. However we point out on the top of the related work that for relatively low traffic values-i.e., when the demands bitrate is lower than at least half the channel linerate-the packet switching solution is more power-efficient, thanks to the opportunity of exploiting traffic grooming to better utilize network resources.

Our key finding is that an increase in the network node count does not consistently increase the power savings of circuit switching over packet switching, but is heavily influenced by the mesh degree and (to a minor extent) by the average physical link length. Increasing the network mesh degree produces higher energy benefits for packet switching than for circuit switching, as more power can be saved in intermediate nodes in the former case.

While we have performed a sensitivity study with respect to non-uniform traffic demands, useful further work would consist in extending this with traffic demands originating from actual measurements. Furthermore, should the power consumption of future packet switches become more proportional to the load, it is likely that this will influence the outcome of our comparison. Finally, our routing algorithm is in both switching paradigms based on a shortest-path approach. It would be interesting to see if power-optimized routing (e.g., achieved with solving an ILP problem) changes the trends that we have observed.

\section{ACKNOWLEDGMENTS}

The work described in this paper was carried out with the support of the iMinds-project GreenICT and the European Community's Seventh Framework Programme (FP7/20072013) under grant agreement n. 257740 (Network of Excellence TREND).

\section{REFERENCES}

[1] S. Lambert, W. Van Heddeghem, W. Vereecken, B. Lannoo, D. Colle, and M. Pickavet, "Worldwide electricity consumption of communication networks," Optics Express, vol. 20, pp. B513-B524, December 2012.

[2] C. Lange, D. Kosiankowski, R. Weidmann, and A. Gladisch, "Energy consumption of telecommunication networks and related improvement options," Journal of Selected Topics in Quantum Electronics, vol. 17, no. 2, pp. 285-295, 2011.

[3] J. Baliga, R. Ayre, K. Hinton, W. V. Sorin, and R. S. Tucker, "Energy Consumption in Optical IP Networks," Journal of Lightwave Technology, vol. 27, pp. 2391-2403, July 2009.

[4] G. Shen and R. Tucker, "Energy-minimized design for IP over WDM networks," Journal of Optical Communications and Networking, vol. 1, pp. 176-186, June 2009.

[5] W. Van Heddeghem, F. Idzikowski, W. Vereecken, D. Colle, M. Pickavet, and P. Demeester, "Power consumption modeling in optical multilayer networks," Photonic Network Communications, vol. 24, pp. 86-102, October 2012.

[6] S. Aleksić, W. Van Heddeghem, and M. Pickavet, "Scalability and power consumption of static optical core networks," in Proc. of Globecom, Anaheim, USA, December 2012.

[7] A. Bianco, E. Bonetto, F. Musumeci, A. Pattavina, and M. Tornatore, "CapEx/OpEx evaluation of circuit vs packet switched optical networks," in Proc. of ONDM, Brest, France, April 2013.

[8] S. Aleksić, "Analysis of Power Consumption in Future High-Capacity Network Nodes," Journal of Optical Communications and Networking, vol. 1, pp. 245-258, August 2009.

[9] X. Dong, T. El-Gorashi, and J. Elmirghani, "On the energy efficiency of physical topology design for IP over WDM networks," Journal of Lightwave Technology, vol. 30, pp. 1931-1942, June 2012.

[10] W. Van Heddeghem, F. Idzikowski, E. Le Rouzic, J. Mazeas, H. Poignant, S. Salaun, B. Lannoo, and D. Colle, "Evaluation of power rating of core network equipment in practical deployments," in Proc. of GreenCom online, September 2012.

[11] A. Vishwanath, J. Zhu, K. Hinton, R. Ayre, and R. S. Tucker, "Estimating the Energy Consumption for Packet Processing, Storage and Switching in Optical-IP Routers," in Optical Fiber Communication Conference, 2013.

[12] F. Idzikowski et al., "Final report for the IRA "Energy-efficient use of network core resources," Deliverable D3.3, TREND Project, 2012.

[13] D. Staessens et al., "Value analysis report on the use of the network planning tool," Deliverable D7.3, DICONET, European ICT-research project, 2010. 